\title{
Adequate endoscopic mucosal resection for early gastric cancer obtained from the dissecting microscopic features of the resected specimens
}

\author{
Masao Tani ${ }^{1}$, Kimiya Takeshita ${ }^{2}$, Haruhiro Inoue ${ }^{1}$, and Takehisa Iwai ${ }^{1}$ \\ ${ }^{1}$ First Department of Surgery, Tokyo Medical and Dental University, School of Medicine, 1-5-45, Yushima, Bunkyo-Ku, Tokyo 113-8519, \\ Japan \\ ${ }^{2}$ Department of Endoscopic Diagnosis and Therapy, Tokyo Medical and Dental University, School of Medicine, Tokyo, Japan
}

\begin{abstract}
Background. We have employed endoscopic mucosal resection (EMR), using a cap-fitted panendoscope (EMRC), for early gastric cancer since 1992 . The presence of an adequate surgical margin is a requirement because of the radicality of EMR, and dissecting microscopic examination is useful in regard to the diagnosis of spread of the disease.

Methods. To devise an adequate method of EMR that allows no lateral residue, we examined gastric mucosal specimens obtained by EMRC. One hundred and sixty-seven specimens from 97 lesions in 85 patients treated by EMRC were examined in regard to characteristic features, the recovery of marks made around the lesion, and the frequency of residue, and comparisons were made between the dissecting microscopic and histopathological findings.

Results. The first specimen obtained with a large cap under full suction was a circular shape measuring $21 \times 19 \mathrm{~mm}$. The second specimen from fractionated resection was a half-moon or crescent shape, and the third specimen had a ginkgo leaflike or irregular shape. In the elevated lesions, coincidence regarding the spread, as determined by dissecting microscopy and histopathology, was present in $62(93 \%)$ of the 67 lesions. In $16(53 \%)$ of 30 flat or depressed lesions, there was a difference of 2 to $5 \mathrm{~mm}$ between the spread determined by these two examinations.

Conclusion. It is important to place an adequate number of marks around the lesion and recover all marks by resection. When an elevated lesion measures $15 \mathrm{~mm}$ or more, and a flat or depressed lesion is not clearly demarcated, aggressive use of planned fractionated resection seems to be the best way to prevent a lateral residue in EMR.
\end{abstract}

Key words Early gastric cancer · Endoscopic mucosal resection $\cdot$ Dissecting microscopic examination

Offprint requests to: $\mathrm{M}$. Tani

Received: February 1, 2001 / Accepted: July 3, 2001

\section{Introduction}

Clinicopathological analyses of surgically treated early gastric cancers have revealed that some of them would have been curable by endoscopic treatment.

The main endoscopic treatment currently used for early gastric cancer is endoscopic mucosal resection (EMR), which allows histopathological examination of the resected specimen. In our department, since 1992, we have employed EMR in which we use, a cap-fitted panendoscope (EMRC) [1-4].

Because of the radicality of EMR, the presence of an adequate surgical margin is a requirement. Therefore, in examining the resected specimen, diagnosis of the spread, as well as the malignancy of the disease, is important, and dissecting microscopic examination is useful in this regard [5].

In the present study, we examined the characteristic dissecting microscopic features of gastric mucosal specimens obtained by EMRC in relation to macroscopic type, and diagnosis of the lateral spread of cancer obtained by dissecting microscopic examination was compared with that obtained by histopathological examination. Through this investigation, an appropriate method of mucosal resection that is related to macroscopic type and allows no lateral residue was devised.

Subjects and methods

\section{Subjects}

In this study, we investigated 97 lesions in 85 patients (66 men and 19 women) treated by EMRC and observed by dissecting microscopy between August 1992 and June 1999. The mean age of the men was $66( \pm 9.2)$ and that of the women, $71( \pm 7.1)$ years. The lesions were distributed over the entire stomach. 
Sixty-seven lesions were elevated; 29 of these were adenomas, 3 were type $0 \mathrm{I}$ early cancers and 35 were type 0IIa early cancers. The remaining 30 lesions were flat or depressed, and consisted of 1 adenoma, 3 type 0IIb early cancers, and 26 type 0IIc early cancers.

All technical terms used in this report are in accordance with the Japanese classification of gastric carcinoma (2nd English edition) [6]. The statistical significance of differences was analyzed by Student's $t$-test, with the level of significance set at $5 \%$.

\section{Method of resection}

According to the EMRC procedure reported previously [7], we treated the lesions by single (en-bloc) resection or multiple-fragment (fractionated) resection. Of the 97 lesions, en-bloc resection was indicated for 59 (46 elevated and 13 flat or depressed lesions), while fractionated resection was indicated for the remaining 38 (21 elevated and 17 flat or depressed lesions).

\section{Method of observation}

Each resected specimen was immediately extended and fixed with pins on a rubber board, where it was subjected to morphological observation, twodimensional measurements, and the counting of marks that had been made around the lesion. In the specimens obtained by fractionated resection, the specimens were reconstructed on the basis of a copy of the endoscopic front view of the lesion taken after the lesion was marked prior to resection. After the mucus was washed out, the specimen was double-stained with Alcian blue and hematoxylin, and observed under an Olympus SZH-131 dissecting microscope (Olympus, Tokyo, Japan).

During dissecting microscopy, a magnifying power of 7.5 was used for observing the shape of the specimen, counting of marks within the specimen, and identifying the location of the lesion and its position in relation to the marks. The magnifying power was then increased to 15,30 , and 60 , to observe the border between the lesion and the surrounding normal mucosa. In this way, the malignancy and the spread of the lesion were assessed, and the lesion was photographed with $35 \mathrm{~mm}$ color slide film.

The stainability of the lesion was rated as good if it was stained well overall, poor if staining was generally poor, and irregular if staining occurred in patches.

The pit pattern of the mucosal surface was classified as a foveolar pattern (FP) when there was a regular arrangement of gastric foveolae alone, a sulciolar pattern (SP) when there was a regular pattern of gastric sulculi, or a foveolar/sulciolar pattern (FSP) when there was a mixture of FP and SP [8]. The appearance of the pit pattern was expressed as regular, irregular (e.g., disordered or densely increased), or absent.

Dissecting microscopic observation was carried out by the first author alone. The spread of the lesion was diagnosed comprehensively in terms of the extent of elevation or concavity; the extent of the disordered, densely increased, or absent pit pattern; and the extent of irregular or poor staining with hematoxylin.

The extent of the lesion, as determined by dissecting microscopy, was recorded, and a cut line was determined that was vertical to the tangential line drawn for the surgical cut end closest to the lesion. The specimen was sectioned at 2-mm intervals, and histopathologically examined for histological type, depth of invasion, spread of lesion, and presence or absence of malignancy in the surgical margin.

In the diagnosis of the spread of the lesion, the findings of dissecting microscopy and the results of histopathological mapping were compared, and agreement between the results was expressed as discrepancynegative $(-)$, while disagreement was expressed as discrepancy-positive $(+)$.

Specimens obtained by fractionated resection were also examined to determine the size of the specimen, the recovery of marks, the frequency of successful reconstruction, and the frequency of residue.

\section{Results}

\section{Characteristic features of EMRC specimens}

Fifty-nine specimens were obtained from the 59 lesions subjected to en-bloc resection, and from the 38 lesions subjected to fractionated resection, we obtained 108 specimens (34 specimens by two-fragment resection, 33 by three-fragment resection, 36 by four-fragment resection, 5 by five-fragment resection). In total, 167 specimens were obtained from the 97 lesions.

Morphology of the resected specimens (Table 1). The specimens obtained by en-bloc EMRC or by the first session of single fractionated EMRC were circular in 95 of the 97 lesions, with oblong specimens in two lesions. The second specimens of fractionated resection were half-moon or crescent-shaped, and the third or fourth specimens had ginkgo leaf-like or irregular shapes (Fig. 1).

Size of the resected specimens (Tables 2,3,4). The size of EMRC specimens depended on the size of the cap and the negative pressure applied for suctioning. Table 2 shows the average size of the first specimens resected from the 97 lesions. Application of the maximum negative pressure is expressed as full suction, and that of 
Table 1. Morphology of specimens resected by EMRC

\begin{tabular}{lccc}
\hline & \multicolumn{3}{c}{ Number of specimens } \\
\cline { 2 - 4 } & $\begin{array}{c}\text { First } \\
\text { specimen } \\
n=97\end{array}$ & $\begin{array}{c}\text { Second } \\
\text { specimen } \\
n=38\end{array}$ & $\begin{array}{c}\text { Third, fourth, or } \\
\text { fifth specimen } \\
n=21\end{array}$ \\
Shape of specimen & 95 & & \\
\hline Circular & 2 & 1 & 4 \\
$\begin{array}{l}\text { Oblong } \\
\begin{array}{l}\text { Half-moon or } \\
\text { crescent-shaped }\end{array}\end{array}$ & 36 & 11 \\
$\begin{array}{l}\text { Ginkgo leaf-like } \\
\text { Irregular }\end{array}$ & & & 6 \\
\hline EMRC, Endoscopic mulat & & \\
\hline
\end{tabular}

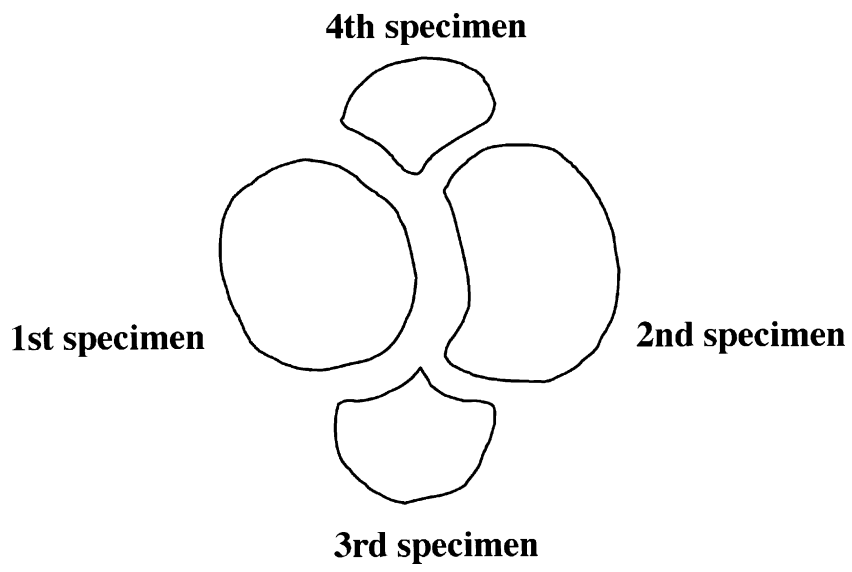

Fig. 1. Morphology of the specimens obtained by endoscopic mucosal resection with cap-fitted panendoscope (EMRC); the first specimen was circular, the second was half-moon or crescent-shaped, and the third or fourth specimen had a ginkgo leaf-like or irregular shape

lighter negative pressure as moderate suction. Specimens obtained using a cap designed for the Olympus EVIS 2T200 under full suction were significantly larger than the other specimens.

When the 64 specimens obtained using the cap for the EVIS 2T200 under full suction were classified by location as in the upper third of the stomach, middle third, or lower third (UML), the size of specimens was generally similar regardless of their location, although there was slight variation within region $\mathrm{U}$ (Table 3).

Among the specimens obtained by fractionated resection, the second specimens showed marked differences between the major axis and minor axis, reflecting their half-moon or crescent shapes (Table 4).

Other characteristic features of resected specimens. Fissures were present in part of the mucosa in $32(19 \%)$ of the 167 resected specimens, and all 167 exhibited congestion within the mucosal layer on histopathological examination.
Table 2. Size of cap, negative pressure applied for suctioning, and average size of EMRC specimen $(\mathrm{mm})$

\begin{tabular}{lcc}
\hline Cap & Full suction & Moderate suction \\
\hline $\mathrm{T}$ & $21.1( \pm 2.7) \times 19.2( \pm 2.8)$ & $16.9( \pm 2.2) \times 15.3( \pm 1.7)$ \\
& $n=64$ & $n=23$ \\
$\mathrm{Q}$ & $15.5( \pm 2.1) \times 14.2( \pm 2.2)$ & \\
& $n=10$ &
\end{tabular}

T, Cap designed for Olympus EVIS 2T200 (inner diameter, $13.5 \mathrm{~mm}$; depth, $10 \mathrm{~mm}$ ); Q, cap designed for Olympus EVIS Q200 (inner diameter, $11.5 \mathrm{~mm}$; depth, $10 \mathrm{~mm}$ )

Table 3. Location and average size of EMRC specimens obtained using cap for Olympus EVIS 2T200 under full suction ${ }^{\mathrm{a}}$

\begin{tabular}{|c|c|c|}
\hline Location & Size of specimen $(\mathrm{m}$ & \\
\hline $\mathrm{U}$ & $21.9( \pm 4.2) \times 19.6( \pm 4.3)$ & $n=9$ \\
\hline M & $21.0( \pm 2.2) \times 19.4( \pm 2.2)$ & $n=24$ \\
\hline $\mathrm{L}$ & $21.0( \pm 2.4) \times 19.0( \pm 2.6)$ & $n=31$ \\
\hline
\end{tabular}

U, Upper third of the stomach; M, middle third of the stomach; $\mathrm{L}$, lower third of the stomach

"See text for definition of "full suction"

Table 4. Average size of EMRC specimens obtained by fractionated resection

\begin{tabular}{|c|c|c|}
\hline Specimen & \multicolumn{2}{|c|}{ Size of specimen $(\mathrm{mm})$} \\
\hline First & $19.6( \pm 2.8) \times 17.6( \pm 2.7)$ & $n=38$ \\
\hline Second & $17.4( \pm 3.3) \times 11.6( \pm 3.3)$ & $n=17$ \\
\hline Third & $14.9( \pm 3.3) \times 11.5( \pm 3.4)$ & $n=11$ \\
\hline Fourth & $15.1( \pm 6.0) \times 10.7( \pm 3.9)$ & $n=9$ \\
\hline
\end{tabular}

Size of specimen, recovery of marks, frequency of successful reconstruction, and frequency of residue in fractionated resections

Elevated lesions (Table 5). Of the 21 elevated lesions subjected to fractionated resection, 1 lesion was resected without marking, another was resected after the placement of one mark, and 19 were resected after the placement of four to ten circumferential marks. The lesion resected without marking was able to be reconstructed because its elevation was distinct. The lesion resected with one mark was small and did not contain the mark; thus, reconstruction was not possible. All marks were recovered in 12 of the 19 lesions with circumferential marking, whereas some marks were not recovered in the remaining 7 lesions. In 1 of these 7 lesions, the lesion could not be reconstructed because the specimens were small and the number of marks insufficient. No residual cancer was found in any cases.

Flat or depressed lesions (Table 6). Of the 17 flat or depressed lesions subjected to fractionated resection, 1 lesion was resected without marking, and 16 lesions 
Table 5. Size of specimen, recovery of marks, frequency of successful reconstruction, and frequency of residue in fractionated resection of elevated lesions

\begin{tabular}{|c|c|c|c|c|c|c|c|c|}
\hline \multirow{2}{*}{$\begin{array}{l}\text { Size of } \\
\text { lesion } \\
(\mathrm{mm})\end{array}$} & \multirow{2}{*}{$\begin{array}{l}\text { No. of } \\
\text { marks }\end{array}$} & \multicolumn{5}{|c|}{ No. of recovered marks in the specimen } & \multirow[b]{2}{*}{ Reconstruction } & \multirow[b]{2}{*}{ Residue } \\
\hline & & $1 \mathrm{st}$ & 2nd & $3 r d$ & 4 th & 5 th & & \\
\hline 12 & 6 & $2(14)$ & $3(12)$ & $1(10)$ & & & $\bigcirc$ & - \\
\hline 14 & 4 & $3(21)$ & $1(17)$ & & & & $\bigcirc$ & - \\
\hline 20 & 10 & $4(20)$ & $4(21)$ & $1(14)$ & $1(10)$ & & $\bigcirc$ & - \\
\hline 17 & 8 & $3(19)$ & $2(18)$ & & & & $\bigcirc$ & - \\
\hline 25 & 0 & $0(22)$ & $0(22)$ & & & & $\bigcirc$ & - \\
\hline 18 & 6 & $3(16)$ & $2(16)$ & & & & 0 & - \\
\hline 13 & 7 & $1(18)$ & 0 (13) & $1(10)$ & $0(6)$ & & $\times$ & - \\
\hline 8 & 1 & 0 (12) & $1(6)$ & & & & $\times$ & - \\
\hline 16 & 5 & $2(22)$ & $2(20)$ & $1(18)$ & & & 0 & - \\
\hline 25 & 5 & $1(18)$ & $1(15)$ & $2(16)$ & $1(16)$ & & 0 & - \\
\hline 15 & 4 & $1(24)$ & $2(16)$ & $1(12)$ & & & 0 & - \\
\hline 15 & 8 & $4(23)$ & $2(20)$ & $2(17)$ & & & 0 & - \\
\hline 16 & 9 & $3(18)$ & $2(18)$ & 1 (14) & $1(10)$ & & $\bigcirc$ & - \\
\hline 16 & 9 & 3 (19) & 2 (16) & $1(14)$ & $2(17)$ & 1 (17) & 0 & - \\
\hline 13 & 6 & $3(20)$ & $3(20)$ & 0 (10) & & & 0 & - \\
\hline 24 & 10 & $5(25)$ & $3(20)$ & $1(18)$ & $1(20)$ & & 0 & - \\
\hline 14 & 10 & $6(20)$ & $2(22)$ & & & & 0 & - \\
\hline 18 & 9 & $3(20)$ & 3 (18) & $2(22)$ & 1 (18) & & 0 & - \\
\hline 23 & 13 & $6(22)$ & $5(20)$ & $1(20)$ & & & 0 & - \\
\hline 10 & 5 & $2(22)$ & $1(20)$ & & & & 0 & - \\
\hline 17 & 4 & $3(20)$ & 1 (19) & & & & $\bigcirc$ & - \\
\hline
\end{tabular}

Numbers in parentheses show the size of the specimen (in $\mathrm{mm}$ )

Circles, Successful reconstruction; Crosses, unsuccessful reconstruction; -, negative for residue

Table 6. Size of specimen, recovery of marks, frequency of successful reconstruction, and frequency of residue in fractionated resection of flat or depressed lesions

\begin{tabular}{|c|c|c|c|c|c|c|c|c|}
\hline \multirow{2}{*}{$\begin{array}{l}\text { Size of } \\
\text { lesion } \\
(\mathrm{mm})\end{array}$} & \multirow{2}{*}{$\begin{array}{l}\text { No. of } \\
\text { marks }\end{array}$} & \multicolumn{5}{|c|}{ No. of recovered marks in the specimen } & \multirow[b]{2}{*}{ Reconstruction } & \multirow[b]{2}{*}{ Residue } \\
\hline & & $1 \mathrm{st}$ & 2nd & $3 r d$ & 4th & 5 th & & \\
\hline 25 & 7 & $0(23)$ & $0(16)$ & $0(15)$ & & & $\times$ & - \\
\hline 10 & 0 & $0(20)$ & $0(18)$ & & & & $\bigcirc$ & - \\
\hline 7 & 9 & $3(17)$ & $3(17)$ & $2(16)$ & $1(16)$ & & $\bigcirc$ & - \\
\hline 11 & 4 & $3(20)$ & $1(12)$ & & & & $\bigcirc$ & - \\
\hline 14 & 6 & $2(21)$ & $2(18)$ & & & & $\bigcirc$ & - \\
\hline 12 & 4 & $3(17)$ & $1(14)$ & & & & $\bigcirc$ & - \\
\hline 30 & 6 & $1(25)$ & $0(25)$ & $0(15)$ & 0 (18) & & $\times$ & + \\
\hline 13 & 10 & $5(22)$ & 4 (19) & & & & $\bigcirc$ & - \\
\hline 12 & 9 & 4 (18) & 3 (18) & $1(10)$ & & & $\bigcirc$ & - \\
\hline 5 & 7 & $2(15)$ & $3(15)$ & 1 (14) & $1(10)$ & & $\bigcirc$ & + \\
\hline 9 & 4 & $2(18)$ & $2(18)$ & & & & 0 & + \\
\hline 13 & 9 & 2 (18) & 2 (18) & $4(17)$ & & & 0 & - \\
\hline 13 & 10 & 4 (19) & $4(18)$ & $2(17)$ & & & $\bigcirc$ & - \\
\hline 9 & 8 & $6(20)$ & $2(18)$ & & & & 0 & - \\
\hline 13 & 10 & $7(21)$ & $3(17)$ & & & & $\bigcirc$ & - \\
\hline 10 & 9 & $5(17)$ & $3(15)$ & $1(13)$ & & & $\bigcirc$ & - \\
\hline 10 & 9 & $4(18)$ & $5(18)$ & & & & 0 & - \\
\hline
\end{tabular}

Numbers in parentheses show the size of the specimen (in $\mathrm{mm}$ )

Circles, Successful reconstruction; Crosses, unsuccessful reconstruction; - , negative for residue; + , positive for residue 

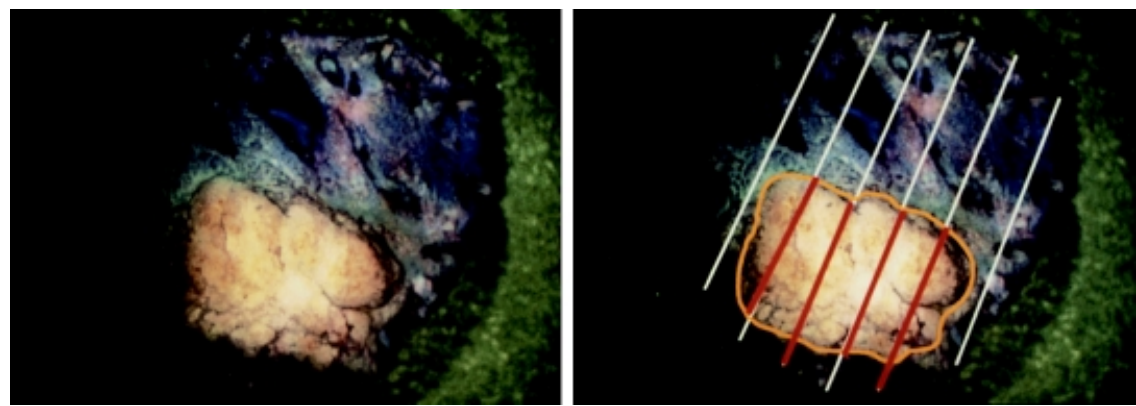

Fig. 2. Elevated gastric adenoma; the white lines are the cut lines, the orange line is the dissecting microscopic spread, and the red lines are the histopathological mapping of the lesion. Although stainability was poor, dissecting microscopy revealed a well maintained sulciolar pattern (SP), and, thus, the poorly stained elevated area was diagnosed as adenoma. Histopathologically, the adenoma had extended to the area of the burn effect in the surgical margin, and the surgical margin was positive for adenoma
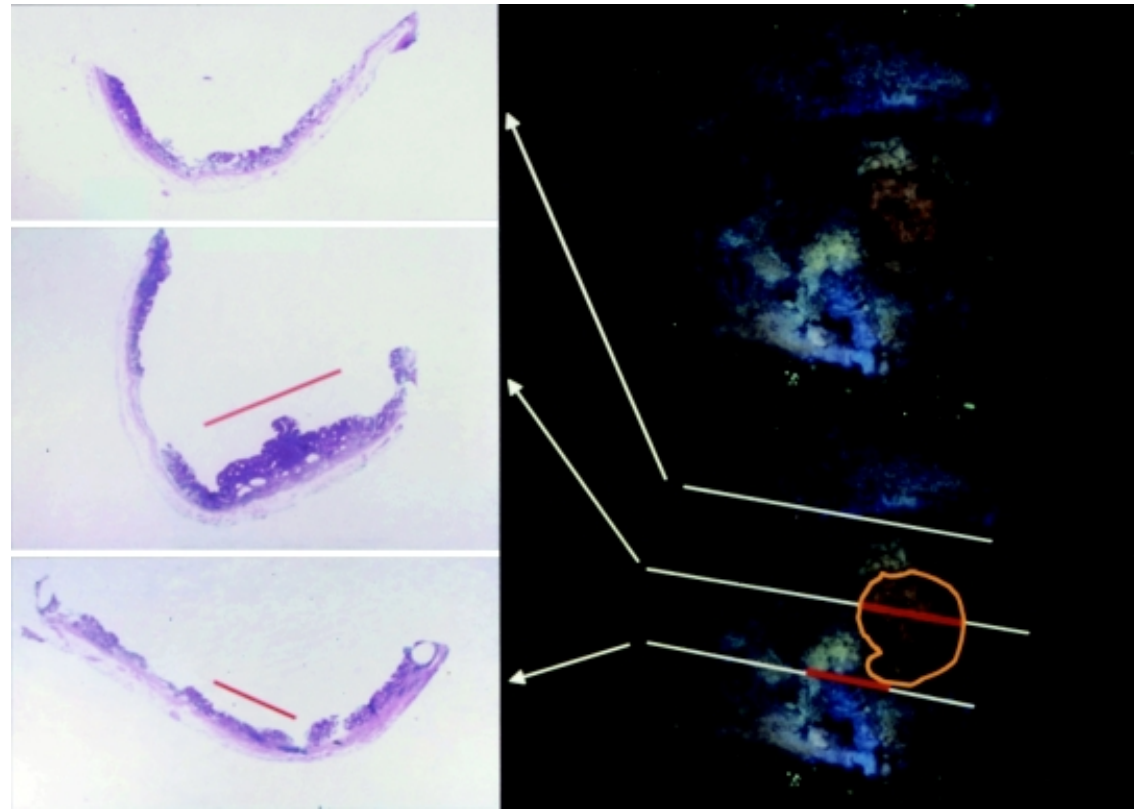

Fig. 3. OIIa-Type early gastric cancer; the white lines are the cut lines, the orange line is the dissecting microscopic spread, and the red lines are the histopathological mapping of the lesion. The 0IIb component surrounding the elevation had been overlooked were resected after the placement of four to ten circumferential marks. The lesion without marking was resected in two fragments, and therefore was able to be reconstructed on the basis of the shapes of the specimens. Although all marks were recovered in 11 of the 16 lesions with circumferential marking, some marks were left unrecovered in 5 lesions, and reconstruction failed in 2 of these 5 because of the absence or insufficiency of marks. There was residue in 3 lesions, which resulted from incorrect diagnosis of the spread of the lesion prior to resection.

\section{Dissecting microscopic observation and histopathological examination of elevated lesions}

In the 67 elevated lesions, coincidence regarding the extent of spread, as determined by dissecting micros- copy and histopathology was present in 62 (93\%). The reason for the discrepancy in 5 lesions was an overlooking of the surrounding flat lesion in 3 lesions and misdiagnosis, owing to the burn effect in the surgical margin, in 2 lesions.

Elevated gastric adenomas. The 29 elevated gastric adenomas measured 5-20 mm. En-bloc resection was applied to 25 of these lesions, and fractionated resection to the remaining 4 lesions. Dissecting microscopic observation revealed variable stainability, but the pit pattern was regular in 20 of the 29 lesions. With regard to the spread of the lesion, the results of diagnosis by dissecting microscopy and histopathological examination were identical in 27 of the 29 lesions, with a difference of $2 \mathrm{~mm}$ between the diagnoses in the other 2 lesions (Fig. 2 ). These discrepancies were a result of the overlooking 
of the modification of the burn effect in the surgical margin and the overlooking of the surrounding flat spread of the adenoma.

OI-Type early gastric cancers. The 3 0I-type early gastric cancers showed irregular stainability, villous unevenness, and clear demarcation from the surrounding normal mucosa on dissecting microscopic examination. There was no discrepancy in the diagnosis of the spread of the lesion between dissecting microscopic diagnosis and histopathological diagnosis.

OIIa-Type early gastric cancers. The 35 0IIa-type early gastric cancers measured 3-35 mm. Eighteen of them were resected en bloc, and the other 17 were subjected to fractionated resection. Dissecting microscopy revealed poor or irregular stainability in 33 of the 35 lesions, with the pit pattern being irregular or absent in 31 of the 35 lesions. The dissecting microscopic diagnosis and histopathological diagnosis of the spread of the lesion were identical in 32 of the 35 lesions, but there were differences of 2,3 , and $4 \mathrm{~mm}$ in three lesions, respectively. These discrepancies resulted from the overlooking of the spread of the surrounding 0IIb lesion in 2 lesions (Fig. 3) and overdiagnosis of the area modified by the burn in the surgical margin in 1 lesion.

\section{Dissecting microscopic observations and histopathological examination of flat or depressed lesions}

In $16(53 \%)$ of the 30 flat or depressed lesions, there was a difference of 2 to $5 \mathrm{~mm}$ between the spread determined by dissecting microscopy and that determined by histopathology.

Depressed gastric adenoma (Table 7). The only depressed gastric adenoma, which measured $25 \mathrm{~mm}$, was removed in three fragments by fractionated resection. Dissecting microscopy revealed irregular stainability and partially irregular SP in the depressed area, leading to a diagnosis of cancer. Histopathologically, however, it was diagnosed as adenoma. Adenoma present in the slightly elevated areas surrounding the concavity had been overlooked.

OIIb-Type early gastric cancers (Table 7). The three 0IIb-type early gastric cancers were $7-16 \mathrm{~mm}$ in diameter. One of them was resected en bloc, and the other two were resected by the fractionated technique. In all three lesions, the lesion could not be confirmed on the specimens immediately after resection, and the spread of the lesion was also difficult to diagnose by dissecting microscopy. In one lesion, dissecting microscopy failed to recognize the disease. In the remaining two lesions, there were differences of 3 and $5 \mathrm{~mm}$ between the results of dissecting microscopic diagnosis and histopathological diagnosis (Fig. 4).

Depressed early gastric cancers (Table 7). The 26 0IIctype early gastric cancers were $1-30 \mathrm{~mm}$ in diameter. Twelve of them were resected en bloc, and the other 14 were removed by fractionated resection. In 2 lesions, dissecting microscopy failed to recognize the disease. In 20 of the remaining 24 lesions, dissecting microsopcy showed poor or irregular stainability. The pit pattern was irregular or no longer present in 23 of the 24 lesions. The results of diagnosis of the spread of the lesion by dissecting microscopy and histopathological examination were in agreement in 14 of the 26 lesions (Fig. 5), with differences found in the other 12 lesions. The discrepancies were explained by failure to recognize the lesion in 2 lesions, failure to diagnose the slight elevation surrounding the concavity as cancer in 2 lesions, overdiagnosis of the slight elevation surrounding the concavity as cancer in 3 lesions, overlooking the surrounding 0IIb spread in 2 lesions, and overlooking the cancer, owing to modification by the burn effect in the surgical margin, in 3 lesions.

\section{Discussion}

Our department (i.e., First Department of Surgery, Tokyo Medical and Dental University) defines the absolute indications for the endoscopic treatment of early gastric cancer based on clinicopathological analyses of early gastric cancers resected surgically [9], which are as follows: (1) lesions diagnosed as mucosal cancer preoperatively, (2) well differentiated cancer, (3) 0IIa type of $20 \mathrm{~mm}$ or less, or nonulcerative 0IIb or 0IIc type of $10 \mathrm{~mm}$ or less.

After EMR for early gastric cancer, a major concern is cancer residue, caused by incomplete resection. Therefore, to accomplish curative treatment by EMR, it is necessary to resect the lesion with sufficient surgical margins in both the lateral and vertical directions. In the present study, an appropriate method of resection to prevent cancer residue in the lateral direction was considered in relation to macroscopic type, by comparing the characteristic features of EMRC specimens, dissecting microscopic findings, and histopathological findings.

Morphological features of specimens obtained by EMRC depend on the size of the cap used and the negative pressure applied. Specimens obtained with a large cap under full suction almost always had a round shape, and measured $21 \times 19 \mathrm{~mm}$, regardless of the gastric portion where the lesion was located. This technique is disadvantageous in that: (1) the mucosa may occasionally be torn when it is drawn into the cap under 


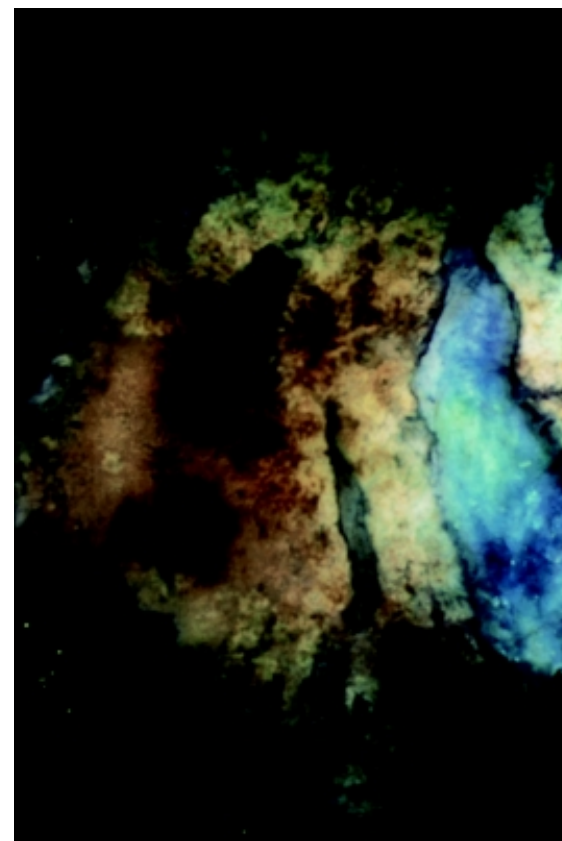

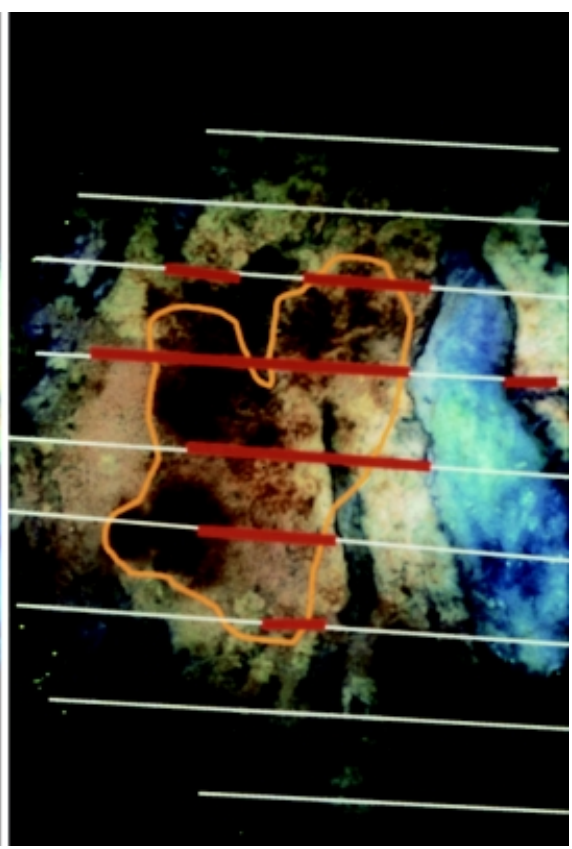

Fig. 4. 0IIb-Type early gastric cancer; the white lines are the cut lines, the orange line is the dissecting microscopic spread, and the red lines are the histopathological mapping of the lesion. Dissecting microscopy showed a well maintained foveolarsulciolar pattern (FSP), and the area with slightly disordered FSP and the surrounding areas of poor stainability were diagnosed as cancer. However, histopathological examination showed malignancy in areas about $5 \mathrm{~mm}$ greater than the extent diagnosed by dissecting microscopy, and severe congestion in the mucosal layer. The surgical margin was negative for cancer
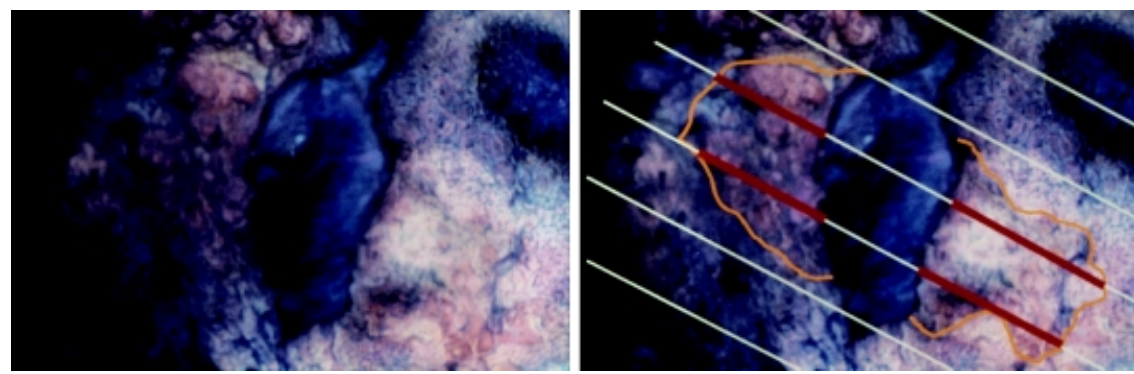

Fig. 5. 0IIc-Type early gastric cancer; the white lines are the cut lines, the orange line is the dissecting microscopic spread, and the red lines are the histopathological mapping of the lesion. Absence of malignancy in the surrounding normal mucosa was ensured by targeting biopsy. After marking was done with reference to the biopsy sites, the lesion was re-

negative pressure, and (2) congestion occurs in the mucosal layer of the specimen. Although these disadvantages cause few problems with elevated lesions, they may make it difficult to diagnose the spread of flat or depressed lesions.

Considering the theoretical extent of EMRC resection in terms of fractionation, ideal overlapping of two circles with diameters of $20 \mathrm{~mm}$ allows resection of an ellipse that has a major axis of $30 \mathrm{~mm}$ and a minor axis of $17 \mathrm{~mm}$, and ideal overlapping of three circles with diameters of $20 \mathrm{~mm}$ allows the resection of a circle with a diameter of $23 \mathrm{~mm}$ (Fig. 6). A single fractionated resection of an extensive area according to this schema is called planned fractionated resection (Fig. 7) [10].

Planned fractionated resection involves fractionation of a marked area containing the lesion, rather than frac- moved by two-fragment planned fractionated resection. Dissecting microscopy showed irregular and partially lost SP with poor stainability in the area consistent with the concavity, and the area was diagnosed as cancer. The extent diagnosed by dissecting microscopy was consistent with the findings of histopathological diagnosis

tionation of the lesion itself. Therefore, marking is a key issue in planned fractionated resection. In our analysis of specimens obtained by fractionated resection in the present study, reconstruction of the specimen was not possible when a small specimen contained no marks or only a single mark. In addition, only one of six marks was recovered in one of three lesions with cancer residue after fractionated resection. For reconstruction of the lesion and prevention of residue, it is important to place an adequate number of marks around the lesion and to recover all the marks by resection.

The use of clips has been reported [11,12] as a better method of reconstruction in fractionated resection. In fractionated resection by the EMRC procedure, the first specimen is circular, and the second specimen has a half-moon or crescent shape, with the concavity facing 


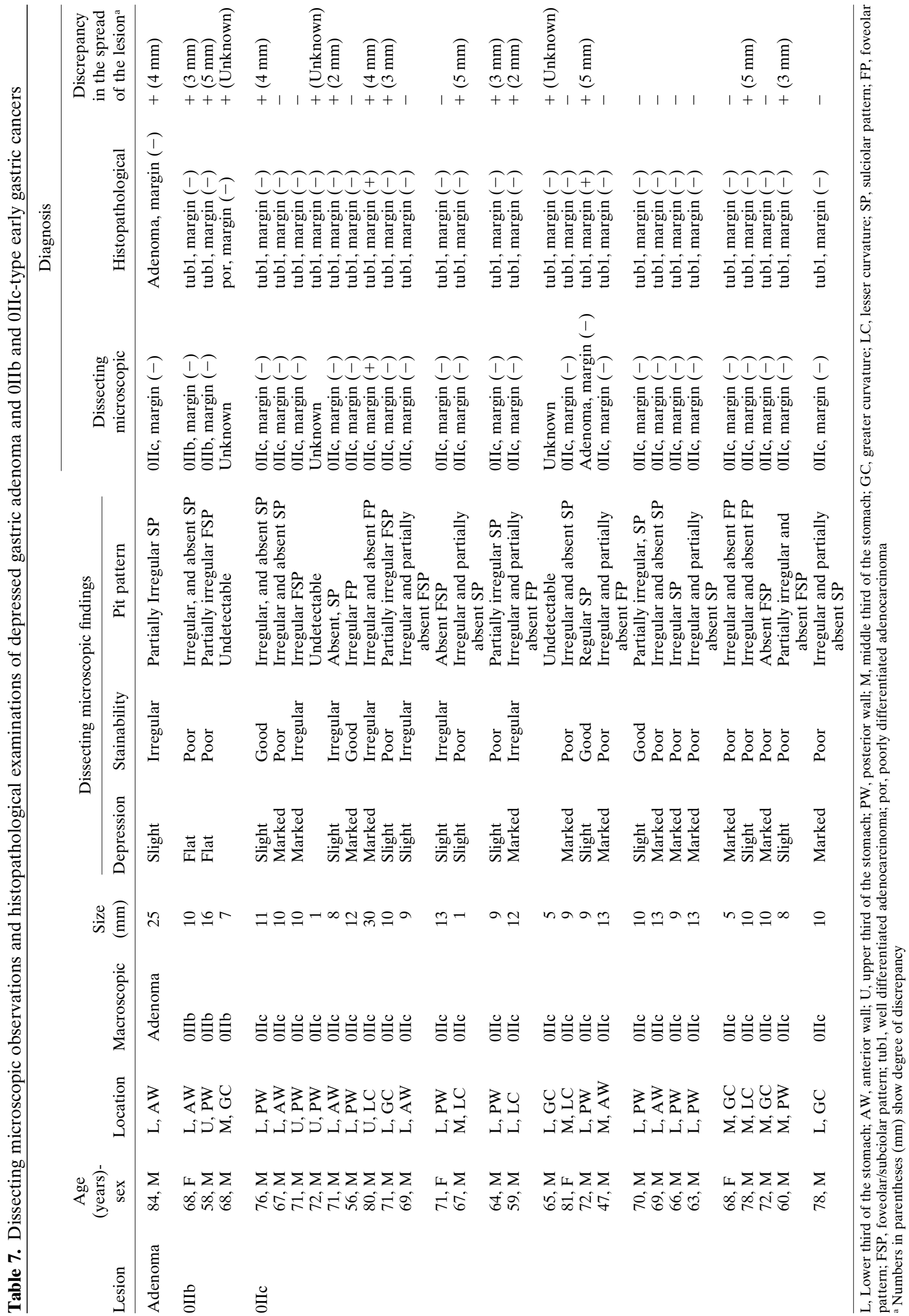




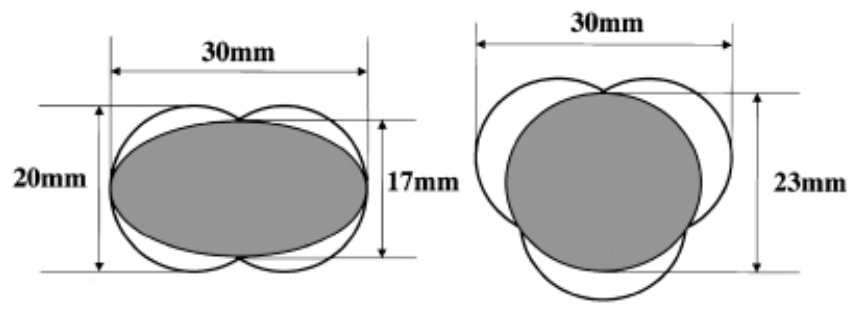

Fig. 6. Theoretical extent of EMRC resection in terms of fractionation

\section{Elevated lesion}
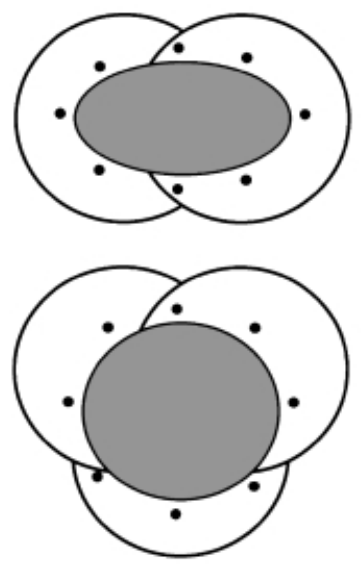

Fig. 7. Schema of planned fractionated resection by EMRC procedure. Gray ellipses, Lesions; dots, marking

the ulcer area made by the first resection, and with the arc made on the opposite side by the resecting line. By collating the marks on the two specimens with those on the lesion in a photograph taken prior to resection, the positional relation of the two specimens becomes apparent, enabling reconstruction. The third specimen has a ginkgo leaf-like shape. By determining the direction against the first and second specimens and by collating the marks, the positional relation of the third specimen becomes apparent.

Sugano et al. [13] reported that, in the diagnosis of the extent of infiltration of flat or depressed early gastric cancer by electronic endoscopy, $78 \%$ of misdiagnosed lesions had obscure borders of the concavity and 56\% contained the IIb component. With EMR specimens from flat lesions or ill demarcated depressed lesions, it is often difficult to diagnose the presence and spread of the lesion macroscopically, because the procedure does not allow on-site determination of whether malignancy is present in the surgical margin. Dissecting microscopic findings occupy a position between macroscopic findings and histopathological findings, enabling us to make a more accurate diagnosis of the malignancy and spread of lesions.
Yoshii [14-16] reported the following characteristic features of dissecting microscopic findings of the mucosa in gastric cancer: disordered, obscured, or absent FP or SP; irregular or poor stainability of the mucosa with hematoxylin; and the appearance of villous unevenness. Yoshii [14-16] also stated that the borders between the noncancerous and cancerous mucosae were generally clear in 0IIa-type lesions, variable on 0IIc-type lesions (because some lesions were surrounded by the 0IIa-type component and some were surrounded by the 0IIb-type component), and extremely difficult to determine in 0IIb-type lesions. In the present study, in the lesions showing a difference between the spread of the lesion as diagnosed by dissecting microsopcy and histopathological examination, the misdiagnosed surrounding components were 0IIb- or 0IIc-types.

Nakamura [17], reporting from the pathological standpoint, reported that, in lesions characterized by artificial destruction of tissue or located near the cut end of the resected specimen, it was difficult to determine the spread of the lesion.

In the present study, five lesions were associated with a discrepancy between the dissecting microscopic and histopathological diagnoses of the extent of the lesion, because of the burn effect in the surgical margin. Adenoma was overlooked in one elevated lesion removed by en-bloc resection; overdiagnosis occurred in one elevated lesion resected by planned fractionated resection; and the presence of cancer was overlooked in three depressed lesions resected by planned fractionated resection. Planned fractionated resection seems to be more likely to cause the burn effect, resulting in diagnostic discrepancy, because the resection line often runs in the vicinity or border of the lesion.

In the elevated lesions, 93\% showed coincidence regarding the extent of spread as determined by dissecting microscopy and histopathology, and it seems apparent that sufficient surgical margins were secured by marking the intact mucosa adjacent to the endoscopic border of the lesion and resecting the mucosa to contain all marks. In contrast, in $53 \%$ of the flat or depressed lesions, there was a difference of 2 to $5 \mathrm{~mm}$ between the spread determined by the two diagnostic procedures. Therefore, surgical margins are not sufficient unless the circumferential marking is at least $5 \mathrm{~mm}$ outside the endoscopic border of the lesion, and an extensive area of mucosa containing the marks is resected.

Thus, in relation to macroscopic type, an appropriate method for endoscopic mucosal resection of early gastric cancer using the EMRC procedure can be summarized as follows.

Elevated lesions measuring $10 \mathrm{~mm}$ or less should be subjected to en-bloc resection after marking is done to indicate the presence of the lesion. Suctioning power at the time of resection should be moderate for lesions of 

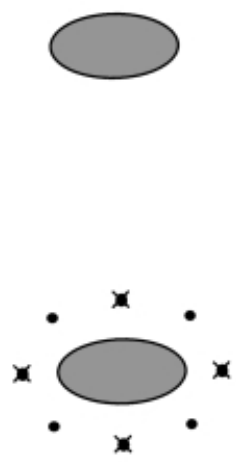

c

Fig. 8a-d. Planned fractionated resection for flat or depressed lesion after targeting biopsy. a Flat or depressed lesions with unclear demarcation. b Targeting biopsy of the surrounding, apparently normal mucosa is performed at four or more sites, each about $5 \mathrm{~mm}$ distant from the border of the lesion, prior to EMR. c Eight or more marks, with reference to the biopsy sites, are placed circumferentially around the lesion. d Extensive area of the mucosa containing the marks is resected. Gray elliptical areas, Lesions; crosses, biopsy sites; dots, marks

about $5 \mathrm{~mm}$ and full for those of about $10 \mathrm{~mm}$. Elevated lesions measuring about $15 \mathrm{~mm}$ or more should be resected in two fragments by planned fractionated resection when they are elliptical and in three fragments by planned fractionated resection when they are circular or nearly circular.

Flat or depressed lesions should be subjected to targeting biopsy of the surrounding, apparently normal mucosa at four or more sites $5 \mathrm{~mm}$ distant from the border of the lesion prior to EMR, and the biopsied sites of normal mucosa should be recorded. For resection, marks with reference to the biopsy sites should be placed circumferentially around the lesion, keeping a distance of at least $5 \mathrm{~mm}$ from the border of the lesion, and an extensive area of the mucosa containing the marks should be resected (Fig. 8).

When an elevated lesion measures approximately $15 \mathrm{~mm}$ or more, and a flat or depressed lesion is not clearly demarcated, aggressive use of planned fractionated resection seems to be the best way to provide an adequate surgical margin and, thus, to prevent lateral residue.

\section{References}

1. Inoue $\mathrm{H}$, Endo $\mathrm{M}$, Takeshita $\mathrm{K}$, Nagahama $\mathrm{Y}$, Yoneshima $\mathrm{H}$, Yoshino K. Endoscopic esophageal mucosal resection using a cap-fitted panendoscope (EMRC) (in Japanese with English abstract). Gastroenterol Endosc 1992;34:2387-90.

2. Inoue H, Takeshita K, Hori H, Muraoka Y, Yoneshima H, Endo M. Endoscopic mucosal resection with a cap-fitted panendoscope for esophagus, stomach, and colon mucosal lesions. Gastrointest Endosc 1993;39:58-62.

3. Takeshita K, Tani M, Inoue H, Saeki I, Honda T, Kando F, et al. A new method of endoscopic mucosal resection of neoplastic lesions in the stomach: its technical features and results. Hepatogastroenterology 1997;44:1602-11.

4. Takeshita K, Tani M, Inoue H, Saeki I, Hayashi S, Honda T, et al. Endoscopic treatment of early esophageal or gastric cancer. Gut 1997;40:123-7.

5. Tada M, Karita M, Yanai H, Kawano H, Takemoto T, Evaluation of endoscopic strip biopsy therapeutically used for early gastric cancer (in Japanese with English abstract). Stomach and Intestine 1988;23:373-85.

6. Japanese Gastric Cancer Association. Japanese classification of gastric carcinoma. 2nd English ed. Gastric Cancer 1998;1:1024.

7. Tani M, Inoue H, Ashikawa T, Kando F, Saito N, Takeshita K, et al. Endoscopic mucosal resection using a cap-fitted panendoscope (EMRC) for gastric tumorous lesion (in Japanese with English abstracts). Prog Dig Endosc 1994;44:73-6.

8. Yoshii T. Patterns of intestinal metaplasia of the gastric mucosa (in Japanese with English abstracts). Stomach and Intestine 1971;6:881-8.

9. Honda T, Takeshita K, Sunagawa M, Ashikawa T, Watanuki S, Tani M, et al. Indication of curative endoscopic therapy for early gastric cancer from the clinicopathological and endoscopic points of view (in Japanese with English abstract). Prog Dig Endosc 1993;42:16-21.

10. Tani M, Inoue H, Kando F, Saito N, Takeshita K, Endo M. Endoscopic mucosal resection for gastric cancer: usefullness of planning fractionated resection (in Japanese with English abstract). Prog Dig Endosc 1995;47:64-8.

11. Inatsuchi S, Tanaka M. Clinical evaluation of an improved technique in strip biopsy for gastric lesion (in Japanese with English abstract). Gastroenterol Endosc 1994;36:939-48.

12. Motohashi O, Ohba K, Sano H, Takagi S, Kiyohashi A, Saigenji K. Multi-EMRL and reconstruction of the resected specimens: experimental study for the purpose of establishing reliable technique and it's clinical use (in Japanese with English abstract). Gastroenterol Endosc 1996;38:2840-47.

13. Sugano $S$, Ohida $M$, Yamagata $S$, Imaizumi $H$, Tanabe $S$, Koizumi W, et al. Diagnosis of electronic endoscopy for the extent of infiltration in the depressed type early gastric cancer (in Japanese with English abstract). Gastroenterol Endosc 1996;38: 2840-47.

14. Yoshii T. Dissecting-microscopic investigation with Alcian bluehematoxylin stain (AH method) of the gastric mucosal surface (3) IIc-type early gastric cancer (in Japanese). Stomach and Intestine 1971;6:1568.

15. Yoshii T. Dissecting-microscopic investigation with Alcian bluehematoxylin stain (AH method) of the gastric mucosal surface (4) IIa-type early gastric cancer (in Japanese). Stomach and Intestine 1971;6:1740.

16. Yoshii T. Dissecting-microscopic investigation with Alcian bluehematoxylin stain (AH method) of the gastric mucosal surface (5) IIb-type early gastric cancer (in Japanese). Stomach and Intestine 1972;7:88

17. Nakamura K. Partial resection of gastric mucosa: evaluation from pathological point of view (in Japanese with English abstract). Stomach and Intestine 1988;23:411-7. 